\title{
ABSOLUTE UV CALIBRATION OF ROCKET PHOTOMETERS USED TO UP-DATE THE OAO CALIBRATION
}

\author{
ALBERT GAIDE
}

Space Astronomy Laboratory, Astronomy Department, University of Wisconsin, Madison, Wisc., U.S.A.

Accurate rocket observations are needed to estimate the post-launch sensitivity of the Wisconsin OAO instruments and to set the absolute scale of the OAO broad-band photometry and spectrophotometry. Seven broad-band photometers, equipping a pointable Aerobee rocket, are calibrated against a source of synchrotron radiation in an attempt to determine, as accurately as possible, the absolute energy distribution of OAO reference stars. The first flight (July 1st, 1970) failed for technical reasons but the experiment will be repeated in 1971 .

\section{Description of the Instrumentation}

Two types of instruments are flown:

(a) $4^{\prime \prime}$ reflectors equipped with an $f / 2.5$ spherical mirror, $\left(\mathrm{Al} /+\mathrm{MgF}_{2}\right)$ coated, a circular diaphragm $\left(1^{\circ}\right)$, a Calcium Fluoride Fabry lens and a 541 Ascop photomultiplier.

(b) $2^{\prime \prime}$ reflectors equipped with an $f / 2.5$ plano-convex Suprasil lens, a circular diaphragm $\left(2^{\circ}\right)$, a Fabry lens, $\mathrm{Al}, \mathrm{MgF}_{2}$ interference filters and a 6256a EMI photomultiplier.

The bands (Table I) are chosen such as to match the bands of the OAO photometers. Each photometer is equipped with its own high-voltage power supply and with an automatic ranging, feed-back type, electrometer (dynamical range of 2.5 decades) designed by $\mathrm{D}$. Michaelski. The telescopes are mounted parallel to the axis of an Aerobee rocket equipped with a stellar pointing system.

TABLE I

Approximative effective wavelength and half width of the photometers

\begin{tabular}{|c|c|c|c|c|}
\hline Collector & Filter & & & Detector \\
\hline $4^{\prime \prime}$ mirror & None & $1350 \AA$ & $200 \AA$ & Ascop $541 \mathrm{~J}$ \\
\hline $4^{\prime \prime}$ mirror & None & $1350 \AA$ & $200 \AA$ & Ascop $541 \mathrm{~J}$ \\
\hline 4" mirror & $\mathrm{Al}+\mathrm{MgF}_{2}$ Interf. filter & 1550 & 250 & Ascop $541 \mathrm{G}$ \\
\hline 2" Quartz lens & $\mathrm{Al}+\mathrm{MgF}_{2}$ Interf. filter & 1950 & 400 & EMI $6256 a$ \\
\hline 2" Quartz lens & $\mathrm{Al}+\mathrm{MgF}_{2}$ Interf. filter & 2000 & 300 & EMI 6256a \\
\hline 2" Quartz lens & $\mathrm{Al}+\mathrm{MgF}_{2}$ Interf. filter & 2400 & 300 & EMI 6256a \\
\hline 2" Quartz lens & $\mathrm{Al}-\mathrm{MgF}_{2}$ Interf. filter & 2900 & 400 & EMI 6256a \\
\hline
\end{tabular}




\section{Calibration Procedure}

The photometers are primarily calibrated against the synchrotron radiation from the University of Wisconsin's $240 \mathrm{MeV}$ electron storage ring. A vacuum monochromator fed by a Hydrogen lamp is used to determine the relative spectral response. A 16-in. vacuum collimator is used to focus the instruments and to determine the profile of their field of view.

The method developed by T. Fairchild is applied to determine the number of electrons in the storage ring and to deduce the energy distribution of their synchrotron radiation. This function, $\operatorname{SR}(\lambda)$, is related to the absolute spectral sensitivity $S_{\lambda}(X, Z)$ in point $X, Z$ of the entrance pupil and to the output signal of the flight electrometer by the relationships:

$$
\begin{aligned}
\overline{\operatorname{Signal}}(x, z)= & \int \operatorname{SR}(\lambda) \cdot S_{\lambda}(x, z) \mathrm{d} \lambda \\
& \simeq \operatorname{SR}\left(\lambda_{\mathrm{eff}}\right) \mu_{0}(x, z)+\left.\frac{1}{2} \mu_{2}(x, z) \frac{\hat{c}^{2}}{\hat{c} \dot{\lambda}^{2}} \operatorname{SR}(\lambda)\right|_{\lambda_{\mathrm{eff}}} \\
& \simeq \operatorname{SR}\left(\lambda_{\mathrm{eff}}\right) \mu_{0}(x, z)
\end{aligned}
$$

since the curvature of $\mathrm{SR}(\lambda)$ is negligible in the wavelength range considered.

In order to acquire a good knowledge of the photometer sensitivity, the effective wavelength and the zero order band-pass momentum should be determined for a grid of points depending on the uniformity of the mirror reflectivity and the photocathode sensitivity. An appreciable simplification of the calibration procedure is achieved however by assuming that the average signal can be related to the average absolute sensitivity by the relationship:

$$
\left.\overline{\text { Signal }}=\operatorname{SR} \overline{\left(\lambda_{\text {eff }}\right.}\right) \overline{\mu_{0}}
$$

where the average effective wavelength is deduced from the relative spectral sensitivity profiles measured in a few points only.

\section{Calibration Accuracy}

The relative uncertainty over the absolute sensitivity $\mu_{0}$ can be expressed as:

$$
\Delta \bar{\mu}_{0} / \bar{\mu}_{0}=\frac{\Delta(\overline{\text { Signal }})}{\overline{\text { Signal }}}+\frac{\Delta \operatorname{SR}\left(\overline{\lambda_{\text {eff }}}\right)}{\operatorname{SR}\left(\overline{\lambda_{\text {eff }}}\right)}
$$

The contribution of the first right-hand side term is small, about $0.5 \%$, if the average signal is deduced from some forty measurements determined within $3 \%$ each. The contribution of the last term is the statistical combination of the relative uncertainties related to the determination of the energy distribution $(3 \%)$ and the effective wavelength $(3 \%)$. With these figures the relative accuracy of the absolute calibration is of $5 \%$. 


\section{Observational Accuracy}

Other factors and other sources of uncertainty appear however in the determination of the stellar fluxes which can be expressed as:

$$
\text { Star flux }\left(\bar{\lambda}_{\text {eff }}\right)=\text { star signal } / \vec{\mu}_{0} \times \text { coll. area } \times \text { atm TRM } \times \mathrm{FVC}
$$

$$
(2 \%) \quad(5 \%) \quad(1 \%) \quad(1 \%) \quad(1 \%)
$$

since the curvature of the energy distribution is negligible for the wavelength range and the stars (A2-B 3) considered. The profile of the field of view being flat within $1 \%$ no corrections for the star position is envisaged so far. A good accuracy is expected on the signals because of the long observations $(20 \mathrm{~s})$ which can be made on each star. The collecting area is delimited by a diaphragm of well-known aperture located in front of the mirror. The residual atmospheric extinction, expected to be less than $10 \%$ in the worst case, can be determined with a sufficient accuracy $(10 \%)$ by observing the same star at different altitudes.

With the figures quoted below each factor, the relative uncertainty which can be expected on the determination of the stellar fluxes is of $10 \%$.

\section{Discussion}

The figure of $10 \%$ represents a reasonable estimate of the expected data accuracy in as much as the photometers are insensitive to polarization and that no changes in the spectral properties of their components occur between calibration and flight operation. With regard to the latter of these arguments, the photometers are calibrated only ten days before launch and a portable vacuum collimator was built in order to compare the flight instruments with identical reference instruments two days before the flight as well as immediately afterwards. The duplication of some of the instruments, namely the 1350 and $2000 \AA$ photometers is also meant to detect any abnormal change of sensitivity. No estimates of the efficiency of these precautions can be expressed at present but the experience made so far with the portable collimator is very encouraging and appreciable improvements are expected in the near future.

The errors due to the appreciable polarization of the synchrotron radiation were not investigated for the first flight. Tests conducted with photomultipliers only, indicated a very weak sensitivity to polarization. Additional effort should be attempted to check the complete photometers but the operation is not an easy one and requires a fairly complicated remote-controlled equipment.

A substantial confidence in the accuracy of the absolute photometry described here is expected for stars of spectral type close to AO because of the great similarity between their energy distribution and the distribution of the synchrotron radiation folded with the transmission of the storage ring Lithium fluoride window. In such a case, the proportionality factor relating the stellar flux to the calibration flux is wavelength independent and is therefore unaffected by the uncertainties related to the knowledge of the average effective wavelength. The similarity of the energy distribu- 
tions can also reduce considerably the differential effects of scattered light which are likely to have led to appreciable errors in calibration techniques using conventional sources.

\section{DISCUSSION}

A. B. Underhill: Is there any list of recommended stars for standard objects in UV photometry? I would like to suggest commission 44 consider setting up a list of suitable stars accessible to rocket and to satellite instruments.

A. Gaide: The number of observable stars is a very limited one for many reasons (tracking possibility, non variability, etc...) No specific choice has yet been made but there should be no great difficulty coming up with a list of candidate stars. 